\title{
Preface:
}

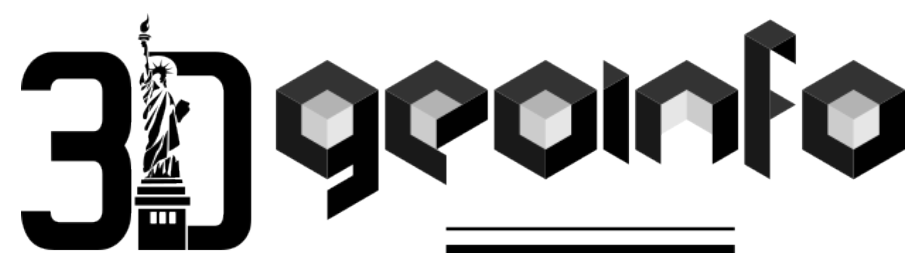

Oct. 11-14, 2021

New York, NY, US

In memory of Martin Isenburg 
Linh Truong-Hong

Department of Geoscience and Remote Sensing, Delft University of Technology, Delft, The

Netherlands

Fengman Jia

Department of Geomatics, University of Calgary, Calgary, Canada

Erzhuo Che

School of Civil and Construction Engineering, Oregon State University, USA

Saeid Emamgholian

Department of Geomatics, Université Laval, Québec, Canada

Debra Laefer

Center for Urban Science and Progress \& Dept. of Civil and Urban Engineering, New York

University, New York, USA

Anh $\mathrm{Vu} \mathrm{Vo}$

School of Computer Science, University College Dublin, Ireland

3D GeoInfo 2021, the $16^{\text {th }}$ GeoInfo conference, is an annual ISPRS workshop offering a forum for leading international decision makers and prominent voices in the field of 3D Geoinformation across the academic, commercial, and public sectors. The 2021 workshop is organized in conjunction with the $7^{\text {th }}$ International FIG workshop on 3D Cadastres. The 2021 event was held virtually. Topics included:

- 3D data creation and acquisition

- 3D data processing and analysis

- 3D data management - data quality, metadata, provenance and trust

- Data integration, information fusion, multi-modal data analysis

- 3D visualization, including gamification, virtual reality, augmented reality

- $\quad 3 \mathrm{D}$ and Artificial Intelligence/Machine Learning

- 3D and Big Data, parallel computing, cloud computing

- 3D city modeling, underground infrastructure modeling, topography, and bathymetry modeling

- Building Information Modeling, Digital Twins, Smart Cities, Smart Infrastructure

- Usability and Human-Computer interaction in 3D GIS

- 3D GIS, spatial analysis and other applications (such as 3D cadastral systems, land administration, utilities, asset management, infrastructure, navigation, urban planning, geology, archaeology, marine systems, simulations, autonomous vehicles, facilities management, energy modeling, disaster and risk management, pandemic monitoring)

The 3D GeoInfo 2021 tracks received 73 manuscripts including 30 full papers and 43 extended abstracts. The manuscripts were reviewed with a double-blind review process by members of the organizing and scientific committee and external reviewers. Ultimately 24 papers were accepted for the ISPRS Annals and 24 papers for the ISPRS Archives. We thank all of the authors and reviewers for their contributions.

We look forward to the 3D GeoInfo 2021 virtual oral presentations and the opportunity to exchange ideas within our workshop and with the presenters and visitors of the $7^{\text {th }}$ International FIG workshop on $3 \mathrm{D}$ Cadastres. 


\section{Organizing Committee:}

Andrea Albright, University of Houston (USA)

Anh $\mathrm{Vu}$ Vo, University College Dublin (Ireland)

Debra Laefer, New York University (USA)

Eftychia Kalogianni, Delft University of Technology (Netherlands)

Erzhuo Che, Oregon State University (USA)

Evan O'Keeffe, New York University (USA)

Fengman Jia, University of Calgary (Canada)

Gene Roe, LiDAR News (USA)

Gerard Dooly, University of Limerick (Ireland)

Hanis Rashidan, University of Technology Malaysia (Malaysia)

Kourosh Khoshelham, University of Melbourne (Australia)

Linh Truong-Hong, Delft University of Technology (Netherlands)

Michael Stanley, New York University (USA)

Nora Gyetvai, Autodesk (USA)

Saeid Emamgholian, Université Laval (Canada)

Sarina Adeli, State University of New York (USA)

Sean Morrish, ESRI (USA)

Shirin Malihi, Technische Universität Braunschweig (Germany)

Siyuan Chen, University College Dublin (Ireland)

Zahiri (Mina) Zohreh, University of Antwerp (Belgium)

Zhe Sun, Carnegie Mellon University (USA)

Zihan Chen, Université Laval (Canada)

\section{Scientific Committee:}

Alias Abdul-Rahman, University Technology Malaysia (Malaysia)

Andreas Georgopolous, National Technical University of Athens (Greek)

Apostolos Arvanitis, Aristotle University of Thessaloniki (Greek)

Baris Kazar, Oracle (USA)

Behnam Atazadeh, University of Melbourne (Australia)

Claire Ellul, University College London (UK)

Claudine Metral, University of Geneva (Swith)

Claudio Persello, University of Twente (Netherlands)

Edward Verbree, Delft University of Technology (Netherlands)

Efi Dimopoulou, National Technical University of Athens (Greek)

Filip Biljecki, National University of Singapore (Singapore)

Francesco Nex, University of Twente (Netherlands)

Hugo Ledoux, Delft University of Technology (Netherlands)

Ismail Rakip Karas, Karabuk University (Turkey)

Ivin Amri Musliman, Universiti Teknologi Malaysia (Malaysia)

Jacynthe Pouliot, Universite Laval (Canada)

Jantien Stoter, Delft University of Technology (Netherlands)

Jose-Paulo de Almeida, Universidade de Coimbra (Portugal)

Kean Huat Soon, Singapore Land Authority (Singapore)

Lars Bodum, Aalborg University (Denmark)

Lars Harrie, Lund University (Sweden)

Leon olde Scholtenhuis, University of Twente (Netherlands)

Lucía Díaz Vilariño, Universidade de Vigo (Spain)

Martin Breunig, Karlsruhe Institute of Technology (Germany)

Martin Kada, Technische Universität Berlin (Germany)

Mike Horhammer, Oracle (USA)

Mila Koeva, University of Twente (Netherlands)

Mohsen Kalantari, University of Melbourne (Australia) 
Peter van Oosterom, Delft University of Technology (Netherlands)

Rudi Stouffs, National University of Singapore (Singapore)

Sagi Dalyot, Israel Institute of Technology (Israel)

Sander Oude Elberink, University of Twente (Netherlands)

Sisi Zlatanova, University of New South Wales (Australia)

Siva Ravada, Oracle (USA)

Thomas Kolbe, Technical University of Munich (Germany)

Umit Isikdag, Mimar Sinan Fine Arts University (Turkey)

Youness Dehbi, University of Bonn (Germany) 